\title{
Analyticity and modulation
}

\section{Broadening the rescale perspective on language logicality}

\author{
Salvatore Pistoia-Reda | ORCID: 0000-0003-3918-214X \\ University Pompeu Fabra Barcelona, Barcelona, Spain \\ salvatore.pistoiareda@upf.edu \\ Uli Sauerland | ORCID: 0000-0003-2175-535X \\ zAS Berlin, Berlin, Germany \\ sauerland@leibniz-zas.de
}

\begin{abstract}
Acceptable analyticities, i.e. contradictions or tautologies, constitute problematic evidence for the idea that language includes a deductive system. In recent discussion, two accounts have been presented in the literature to explain the available evidence. According to one of the accounts, grammatical analyticities are accessible to the system but a pragmatic strengthening repair mechanism can apply and prevent the structures from being actually interpreted as contradictions or tautologies. The proposed data, however, leaves it open whether other versions of the meaning modulation operation are required. Novel evidence we present argues that a loosening version of the repair mechanism must be available. Our observation concerns acceptable lexical contradictions that cannot be rescued if only a strengthening version of the pragmatic strategy is available.
\end{abstract}

\section{Keywords}

language logicality - grammaticality - analyticity - meaning modulation - loosening 
Recent work in linguistics argues for the "logicality" of language. This is the idea that the language system consists of 1 ) a computational device forming complex concepts out of an inventory of basic concepts, and 2) an algorithmic deductive system, i.e. a natural logic, with the ability to draw inferences from these structures. These two components jointly determine the set of usable expressions of natural languages. Chief evidence for this idea comes from the ungrammaticality of some analytic sentences. Standard examples are presented below, from von Fintel (1993) and Chierchia (2013) respectively.

(1) *Someone but John smokes.

(2) *There are any cookies left.

The established account of (1) (Von Fintel, 1993; Gajewski, 2008) is that the restriction by the exceptive but John triggers an obligatory grammatical implicature that the sentence is false without the exceptive. In an acceptable use of an exceptive as in Everyone but John smokes this obligatory implicature derives that not everyone smokes, and therefore predicts the inference that John does not smoke. But in (1), the obligatory implicature corresponds to the proposition No one smokes which combined with the assertion that someone but John smokes amounts to a contradiction. The account of (2) (Chierchia, 2013, ch. I) leads to a contradiction in a similar way, though crucially assuming a controversial extension of the logical vocabulary (see Abrusán, 2019 for relevant discussion). The indefinite any is assumed to generate stronger alternatives. These are obligatorily negated provided that they are not entailed by the prejacent sentence. In a negatively oriented environment as in There aren't any cookies left, alternatives are then not negated and the sentence is shown to be semantically equivalent to the variant without the indefinite. But in (2), alternatives are not entailed by the prejacent. They are consequently obligatorily negated, generating the observed contradiction.

Problematic evidence for the logicality of language assumption comes instead from the grammaticality of sentences that seem to verbalize contradictions in classical logic, as in the examples below (similar data also exist with tautologies). The account is related to work on the interpretation of acceptable contradictions, in particular the type called "borderline contradictions" in Cobreros et al. (2012) (see also Alxatib and Pelletier, 2011; Alxatib et al., 2013). 
(3) It is raining and it is not raining.

(4) John is tall and not tall.

In the literature one finds two alternative explanations for the puzzling behavior, i.e. for the fact that just some analyticities result in ungrammaticality. The standard explanation (Chierchia, 2013; Gajewski, 2009, 2002; Fox and Hackl, 2006; Barwise and Cooper, 1981) argues that the logicality of language assumption should be combined with a modular perspective. This assumes radically unspecified logical forms (which Gajewski refers to as "logical skeletons") whence the contradictions in (3) and (4) cannot be accessed by the mechanism assessing the grammaticality of structures. The observation is that such contradictions are due to the nonfunctional vocabulary, to which the mechanism remains indifferent. Ungrammatical contradictions like (1) and (2) are described as grammatically trivial since, as for instance Chierchia discusses, they remain contradictory for arbitrary substitution of the lexical nodes. Grammatical contradictions like (3) and (4) are instead described as logically trivial since they remain contradictory only for uniform substitution of the lexical nodes. And a mechanism only accessing functional vocabulary is expected to inhibit just grammatical contradictions.

In recent discussion another explanation has been proposed, in Del Pinal (2019) (exploring an idea already expressed in Sauerland, 2014). According to this account, the logicality of language assumption can be maintained while rejecting the modularity vision. In particular, the idea is that the mechanism can access the nonfunctional vocabulary, and can thus see the contradictions in (3) and (4), but a pragmatic repair strategy prevents the structures from being actually interpreted as contradictions via application of a RESCALE operator. The proposal thus crucially builds on the various accounts (Stanley, 2007; Recanati, 2010; Martí, 2006) assuming that a meaning modulation operation (either linguistically triggered by articulated constituents or as an effect of an interpretation function informed by the utterance context) can change the meaning of nonfunctional terms so as to make classical contradictions and tautologies not trivial. Crucially, the strategy is, however, not applicable to functional words and the ungrammatical contradictions in (1) and (2) are unavoidable also on this explanation (see Chierchia, 2019 for possible extensions).

In this paper we investigate the pragmatic repair strategy. In Del Pinal's discussion, a strengthening version of the strategy is provided. Meaning modulation is then obtained as a result of excluding non standard interpretations, via specialization of meanings. As he notes, however, a less constrained, more tolerant version of the strategy is also conceivable and this is assumed to be 
compatible with the spirit of his proposal. In our discussion, we first show that the strengthening strategy works for the proposed examples, i.e. can change the contradictory status of grammatical contradictions but is unable to rescue ungrammatical contradictions, in accordance with our intuitions. Second, we note that the loosening version of the strategy, when suitably defined, can achieve the same result. Then, we submit evidence that could only be explained if the strategy is not restricted to the strengthening sort. In particular, we present sentences that constitute nonfunctional contradictions but are judged as acceptable. As we discuss, however, the strengthening version of the operator cannot change the contradictory status of our cases, which conflicts with our intuitions. We then conclude that the only way to maintain the logicality of language assumption without adopting the modular perspective is to assume that meaning modulation must be loosening in certain occasions.

The rescale perspective on language logicality

Del Pinal's account assumes that the silent operator RESCALE can be inserted into the structure of sentences. Functional and nonfunctional vocabulary are not distinguished for the logicality of language, in difference to the modularity account. But like the modularity account, the rescale account also assumes that only certain analyticities are perceived as ungrammatical.

In the version presented, the operator RESCALE can only apply to lexical terminals and furthermore can only strengthen the interpretation of the terminal, much like the expressions typical or good do. The strengthening version of pragmatic operations like RESCALE are motivated by familiar cases like (5) (Carston, 2002) in which the concept of bachelor does not merely refer to marital status but maybe in addition to eligibility for marriage (e.g. excluding catholic priests). The modulated meaning is obtained via exclusion of logically available interpretations.

(5) Chris wants to meet a bachelor.

Here and in the following, we assume, with Del Pinal, that predicates are automatically given a literal interpretaion, related to what Cobreros et al. (2012) call a "classical" interpretation, i.e. we assume that bachelor is true of any unmarried adult male and raining is true if there is any liquid precipitation. ${ }^{1}$

1 We discuss this assumption further in the conclusion. 
Hence, we assume that the concept raining is also true in more borderline cases of light rain. For example, when REscaLE in the strengthening version Del Pinal presents applies to the concept raining, the resulting interpretation can be paraphrased as raining strongly. The account predicts that (6) should be ungrammatical since it is contradictory, but (7) where RESCALE applies is not contradictory, and it is correclty predicted to be acceptable. Note that the account not only correctly predicts the grammaticality of (6), but furthermore predicts the interpretation of it correctly: It's raining, but not very hard (or in another typical way).

(6) *It is raining and it is not raining

(7) It is raining and it is not RESCALE (raining)

The discussion of this case shows that the REscale operator accounts for grammatical contradictions, but we still need that insertion of RESCALE does not change the contradictory status in the case of ungrammatical contradictions. Consider example (8) of an ungrammatical exceptive similar to the case (1) presented above. We assume that (8) is interpreted on the basis of the logical form in (9) (Gajewski, 2008). The operator $\mathrm{ExH}_{C}$ applied to the prejacent negates all of the alternatives in the set $C$ that are logically stronger than the prejacent. The set $C$ itself is determined by association with focus (Rooth, 1985, 1992), where in particular it associates with the focus on $\neg \operatorname{smart}(x)$. The resulting interpretation of this case is shown in (10).

(8) *Some students but the smart ones smoke.

(9) $\operatorname{ExH}_{C}\left(\exists x\left(\operatorname{student}(x) \wedge[\neg \operatorname{smart}(x)]_{F} \wedge\right.\right.$ smokes $\left.\left.(x)\right)\right)$

(10) $\exists x(\operatorname{student}(x) \wedge \neg \operatorname{smart}(x) \wedge$ smokes $(x) \wedge \neg \exists x(\operatorname{student}(x) \wedge$ smokes $(x))$

Insertion of RESCALE in the representation (9) doesn't affect the contradictory status of (8). If RESCALE is applied to smart as in (11) the contradiction remains.

(11) $\operatorname{ExH}_{C}\left(\exists x\left(\operatorname{student}(x) \wedge[\neg \operatorname{RESCALE}(\operatorname{smart})(x)]_{F} \wedge\right.\right.$ smokes $\left.\left.(x)\right)\right)$

(12) $\exists x(\operatorname{student}(x) \wedge \neg \operatorname{RESCALE}(\operatorname{smart})(x) \wedge \operatorname{smokes}(x)) \wedge \neg \exists x(\operatorname{student}(x)$ $\wedge$ smokes $(x))$ 
If RESCALE applies to student as in (13), crucially this term is interpreted rescaled in both places where it occurs in (14) and therefore the contradiction remains also in this case.

(13) $\operatorname{ExH}_{C}\left(\exists x\left(\operatorname{RESCALE}(\right.\right.$ student $)(x) \wedge[\neg \operatorname{smart}(x)]_{F} \wedge$ smokes $\left.\left.(x)\right)\right)$

(14) $\exists x(\operatorname{Rescale}(\operatorname{student})(x) \wedge \neg(\operatorname{smart})(x) \wedge \operatorname{smokes}(x)) \wedge$

$\neg \exists x(\operatorname{Rescale}($ student $)(x) \wedge$ smokes $(x))$

The invariance of analyticity to the insertion of RESCALE is a consequence of the fact that the logical form structure can be seen as defining the function of predicate meaning in (15). The function yields a contradiction for any $P$ it applies to, with student and RESCALE(student) being two special cases. Crucially the architecture of interpretation in this case blocks RESCALE from applying to the bound occurrences of $P$ in (15).

(15) $\lambda P \exists x(P(x) \wedge \neg(\operatorname{smart})(x) \wedge \operatorname{smokes}(x)) \wedge \neg \exists x(P(x) \wedge$ smokes $(x))$

In his account, Del Pinal already discusses some differences between modularity and rescale accounts (see e.g. Del Pinal 2019: 28-29), and suggests that the latter should be preferred, both philosophically (i.e. in relation to blindness to general knowledge and inferential abilities) and empirically (e.g. with respect to contradictions with variable co-binding of predicates). He also notes that less constrained versions of a rescale account remain possible, other than the one he develops, and that the empirical data he presents leave the account underdetermined in this respect.

\section{Broadening the rescale perspective on language logicality}

\subsection{An alternative loosening rescale: Previous data}

Operations similar to RESCALE, but more liberal, are found in various works in lexical pragmatics. In standard accounts (Carston, 1997; Sperber and Wilson, 2008; Carston, 2002; Wilson and Carston, 2006), ad hoc concepts (Barsalou, $1983,1992)$ are assumed to be created by virtue of two reciprocal pragmatic operations, strengthening and loosening. A weakening version of the RESCALE operator, i.e. RESCALE $\mathrm{loosen}_{\text {, }}$ appears to be motivated by familiar cases like (16). Since the geometric concept hexagonal is not true of the shape of France, the proposal is that RESCALE loosen weakens the concept, similar to the overt roughly or loosely speaking, as shown in (17), and hence (16) is judged true though being "classically" and "strictly" false (Cobreros et al., 2012). 
(16) France is hexagonal.

(17) France is RESCALE loosen $_{\text {(hexagonal) }}$

As we can see, the data discussed in the previous section, concerning the grammaticality of just some analyticities, are compatible with the assumption that RESCALE is either only strengthening or only loosening, as well as with RESCALE being underspecified between RESCALE loosen $_{\text {and }}$ RESCALE strengthen $_{\text {. }}$ Consider how example (6), a grammatical borderline contradiction, is accounted for in a system that has only a loosening operator. As shown in (18), if RESCALE the contradiction isn't resolved since it is impossible that it rains, but doesn't

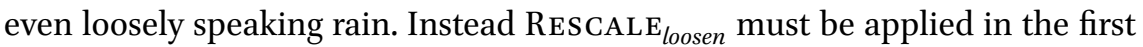
conjunct, as in (19) and the resulting interpretation expresses that it is loosely speaking raining, but not in a stricter sense.

(18) *It is raining and it is not $\operatorname{RescALE}_{\text {loosen }}$ (raining)

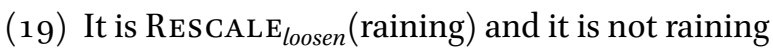

The account of ungrammatical contradictions is crucially unaffected by the change from strengthening to loosening of the operator. Recall that the important feature of that account was that in the ungrammatical cases the logical form could be characterized as a function that applied to any predicate would yield a contradiction. The function (15) yields a contradiction, for the lexical concepts student and $\operatorname{RESCALE}_{\text {strengthen }}\left(\right.$ student), but also for $\operatorname{RESCALE}_{\text {loosen }}$ (student) and any other predicate.

Before we present our evidence, note that an even less restrictive account assumes general meaning modulation as stated in $(20)$. For instance the operator MODULATE freely remaps predicate meanings to other predicate meanings of the same type depending on the context.

(20) $\operatorname{Modulate}_{C}(P)=Q$ for any predicate $Q$ selected by $C$ that is of the same type as $P$

Such free modulation would need to be pragmatically constrained (in ways discussed for instance in Recanati, 2010) since as it stands it make interpretation so liberal that almost any sentence would be true regardless of the facts of the world. Consider, for instance, that (16) with representation (21) would allow the concept of hexagonal to be mapped to either hexagonal or to non-hexagonal, and therefore would be true regardless of the shape of France. 
(21) France is Modulate(hexagonal)

But even this most liberal account predicts ungrammatical contradictions to remain contradictory because the structure of logical form still would constrain MODULATE to apply only to the argument of a function such as (15) that maps any predicate to a contradiction. In the following section, we argue though that other cases shed more light on the nature of the rescale mechanism required.

\subsection{An alternative loosening rescale: New data}

We submit the pair below as new evidence to advance this debate. In particular, we argue that this evidence shows that sometimes RESCALE must be of the loosening sort. This excludes that RESCALE only strengthens the meaning of terms. Empirically, our intuition is that the two sentences are unexpectedly perceived as being grammatical (cf. footnote 2 for a crucial difference between these two cases).

(22) Some female Greek kings come from a warm country.

(23) Some female Greek kings come from a windy city.

Obviously these sentences constitute nonfunctional contradictions under their literal, classical interpretations. The two occurrences of the existential quantifier range over inconceivable entities, making the intersections necessarily empty in every possible world. Del Pinal's account thus assumes that these sentences will be rejected as unnatural unless the proposed pragmatic operator can rescue them by modifying the meaning of the relevant non logical terms. As discussed in the account, formal analyticities are dispreferred if alternative acceptable and potentially informative structures are available. Then, the prediction is that the operator should apply here, in whatever form necessary, to avoid the contradictions. As we mentioned, we believe the submitted acceptability to be evidence that in this case the operator applies to weaken the meaning of the crucial terms. Indeed, if only RESCALE strengthen $_{\text {were available, }}$ an unobserved unacceptability symmetry would actually be predicted between the reported sentences.

We begin by showing that the application of the strengthening version of RESCALE to the above sentences is insufficient. It does not change their contradictory status. In particular, if we applied the operator to one of the two predicates responsible for the generation of the contradictions, i.e. to the predicate female or to the predicate king, we would merely obtain a specification 
of their meanings, which would produce no effect on the contradiction. And

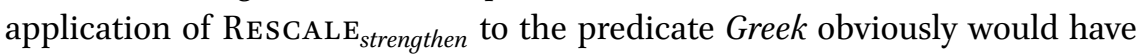
no effect on the generation of the contradiction. Thus, if the operator were limited to RESCALE $E_{\text {strengthen }}$ an unobserved unacceptability symmetry would be expected. This is shown below. Note crucially that, these being scalar sentences, we assume following standard grammatical accounts (Chierchia et al., 2012; Fox, 2007) that the structures include an obligatory exhaustivity operator.

(24) a. $\operatorname{EXH}_{C}\left(\exists x \operatorname{RESCALE}_{\text {strengthen }}(\right.$ female $)$ Greek kings $(x) \wedge$ come from a warm country $(x))$

b. $\operatorname{ExH}_{C}\left(\exists x \operatorname{REsCALE}_{\text {strenghen }}(\right.$ female $)$ Greek kings $(x) \wedge$ come from windy city $(x))$

(25) a. $\operatorname{ExH}_{C}\left(\exists x\right.$ female Greek ResCALE $E_{\text {strengthen }}($ kings $)(x) \wedge$ come from a warm country $(x))$

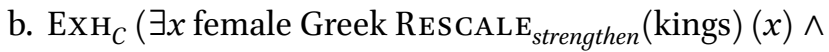
come from windy city $(x))$

(26) a. $\operatorname{ExH}_{C}\left(\exists x\right.$ female RESCALE $E_{\text {strengthen }}($ Greek $)$ kings $(x) \wedge$ come from a warm country $(x))$

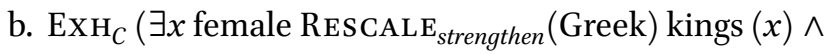
come from windy city $(x))$

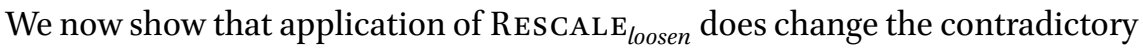
status of sentences. In particular, if applied to the predicate female, this operator remaps the literal content with another predicate which is more tolerant and can be true of male individuals. If applied to the predicate king, the operator remaps the literal content with another predicate which can be true of female individuals. RESCALE at the same time, with a similar result. The following is a possible representation after application of the operator, assuming the first mentioned possible application.

(27) $\operatorname{ExH}_{\mathcal{S}}\left(\exists x \operatorname{ResCALE}_{\text {loosen }}(\right.$ female) Greek kings $(x) \wedge$ come from a warm country $(x))$

(28) $\operatorname{EXH}_{S}\left(\exists x \operatorname{RESCALE}_{\text {loosen }}(\right.$ female $)$ Greek kings $(x) \wedge$ come from a from windy city $(x)$ ) 
Since, as we noted above, the RESCALE account predicts a preference for

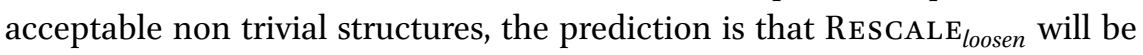
applied in this case to avoid the nonfunctional contradictions. ${ }^{2}$

\section{4}

\section{Final remarks}

In this paper we discussed the assumption that the language system may include a deductive device to draw inferences from the structures. Evidence in favor of this idea comes from the unacceptability of some analytic sentences. However, the literature also presents the case of grammatical analytic sentences. These are crucially nonfunctional analyticities.

In order to explain the puzzling behavior, i.e. the fact that only some analyticities are unacceptable, while at the same time maintaining the logicality of language assumption, two accounts have been presented in the literature. The first account capitalizes on the nonlogical nature of the acceptable analyticities, and assumes that the system assessing grammaticality cannot access the nonfunctional vocabulary, thus being blind to the analytic status of the relevant sentences. In the particular version Gajewski presents, standard logical form representations are substituted with "logical skeletons". The second account rejects the modularity perspective assumed in the first proposal and maintains that the deductive system actually accesses the nonfunctional vocabulary, though a pragmatic strategy then applies to rescue the nonfunctional analyticities in crucial cases. This account builds on proposals concerning contextual meaning modulations.

In this paper we focused on the pragmatic strategy. In the standard account Del Pinal presents, the strategy is described as a strengthening device that specializes the meaning of non logical terms. He also mentions that in principle loosening versions of RESCALE can be conceived without seriously altering the spirit of his proposal, though he does not elaborate. In our discussion, we presented evidence showing that in some cases RESCALE has to be of the loosening sort, otherwise we could not avoid some contradictions to arise. Let us

2 As an additional observation, note that (22) while grammatical is, according to our intuitions,

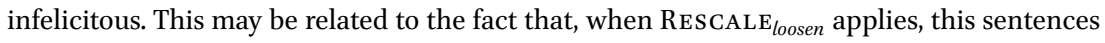
can be reduced to cases where the existential quantifiers range over non existent but conceivable entities, like (i) and (ii) below, first presented in Pistoia-Reda (2017) (see also Magri, 2017).

(i) Some Greek kings come from a warm country.

(ii) Some Greek kings come from a windy city. 
note again that the type of analyticities that require loosening Rescale can be described as lexical (as opposed to formal) analyticities, since their logical status depends on the meaning contribution of content expressions. So clearly a way to maintain a strengthening Rescale is by ignoring lexical analyticities, though we don't think this limitation can be motivated on a pragmatic account à la Del Pinal.

Finally, consider how our conclusions depend on the assumption we introduced in the discussion of example (5), namely that predicates are given "classical" meanings, in the specified sense (Cobreros et al., 2012). Here we did not provide any specific argument in favor of this assumption. So, how would our general argument be affected, though, if we abandon this assumption? Initially it seems that then it would be possible to defend the view that only strengthening is available via RESCALE, against our main point in this paper. For example consider an account of (22) if the basic meaning of female could already be very loose, e.g. so as to include many male individuals. Then, even without assuming the application of rescuing pragmatic strategies, the sentence would not be expected to be ungrammatical, but only predicted to be odd, which is the correct result. However, if a standard loose interpretation is assumed for predicates we would then have to add to this account that most uses of the expression female are interpreted with an application of $\operatorname{RESCALE}_{\text {strengthen }}$, e.g. to prevent a sentence like (29) from having overly strong truth requirements.

(29) This person is not female.

Therefore it seems to us that abandoning the assumption that predicates automatically receive classical interpretations is too costly, and does not seem to support a plausible alternative. We then conclude that the point we raised in this discussion, i.e. that RESCALE must sometimes be of the loosening sort, is not affected.

\section{Acknowledgments}

We gratefully acknowledge support from Generalitat de Catalunya and EU (grant 2018 в о0о7о - Marie Skłodowska-Curie COFUND), DFG (grant SA 925/ 12-2 with SPP 1727 XPrag.de) and Alexander von Humboldt-Stiftung. 


\section{References}

Abrusán, Marta. 2019. Semantic anomaly, pragmatic infelicity, and ungrammaticality. Annual Review of Linguistics 5(1): 329-351.

Alxatib, Sam, Peter Pagin and Uli Sauerland. 2013. Acceptable contradictions: Pragmatics or semantics? A reply to Cobreros et al. Journal of philosophical logic 42(4): 619-634.

Alxatib, Sam and Francis Jeffry Pelletier. 2011. The psychology of vagueness: Borderline cases and contradictions. Mind \& Language 26(3): 287-326.

Barsalou, Lawrence W. 1983. Ad hoc categories. Memory \& Cognition 11(3): 211-227.

Barsalou, Lawrence W. 1992. Frames, concepts, and conceptual fields. In E. Kittay and A. Lehrer (eds), Frames, fields, and contrasts. New essays in semantic and lexical organization, 21-74. Hillsdale NJ: Lawrence Erlbaum Associates.

Barwise, Jon and Robin Cooper. 1981. Generalized quantifiers and natural language. Linguistics and Philosophy 4: 159-219.

Carston, Robyn. 1997. Enrichment and loosening: complementary processes in deriving the proposition expressed? Linguistische Berichte 8:103-127.

Carston, Robyn. 2002. Thoughts and utterances: The pragmatics of explicit communication. Oxford: Blackwell Publishing.

Chierchia, Gennaro. 2013. Logic in Grammar. Polarity, free choice and intervention. Oxford: Oxford University Press.

Chierchia, Gennaro. 2019. On being trivial. Manuscript.

Chierchia, Gennaro, Danny Fox and Benjamin Spector. 2012. Scalar implicature as a grammatical phenomenon. In C. Maienborn, K. von Heusinger and P. Portner. (eds.), Semantics: An International Handbook of Natural Language Meaning, 2297-2331. Berlin: Mouton de Gruyter.

Cobreros, Pablo, Paul Egré, David Ripley and Robert van Rooij. 2012. Tolerant, classical, strict. Journal of Philosophical Logic, 41(2): 347-385.

Del Pinal, Guillermo. 2019. Triviality and logical form. Noûs 53(4): 785-218.

von Fintel, Kai. 1993. Exceptive constructions. Natural Language Semantics 1(2): 123148.

Fox, Danny. 2007. Free choice and the theory of scalar implicatures. In U. Sauerland and P. Stateva, Presupposition and implicature in compositional semantics, 71-120. London: Palgrave Macmillan.

Fox, Danny and Martin Hackl. 2006. The universal density of measurement. Linguistics and Philosophy 29(5): 537-586.

Gajewski, Jon. 2002. L-analyticity and natural language. Manuscript.

Gajewski, Jon. 2008. NPI any and connected exceptive phrases. Natural Language Semantics 16(1): 69-110.

Gajewski, Jon. 20og. L-triviality and grammar. Handout. 
Magri, Giorgio. 2017. Blindness, Short-sightedness, and Hirschberg's contextuallyordered alternatives: A reply to Schlenker (2012). In S. Pistoia-Reda and F. Domaneschi (eds), Linguistic and psycholinguistic approaches on implicatures and presuppositions, 9-54. London: Palgrave Macmillan.

Martí, Luisa. 2006. Unarticulated constituents revisited. Linguistics and Philosophy 29(2): 135-166.

Pistoia-Reda, Salvatore. 2017. Contextual blindness in implicature computation. Natural Language Semantics 25(2): 109-124.

Recanati, François. 2010. Truth-conditional pragmatics. Oxford: Oxford University Press.

Rooth, Mats. 1985. Association with focus. PhD thesis, University of Massachusetts at Amherst.

Rooth, Mats. 1992. A theory of focus interpretation. Natural language semantics 1(1): $75^{-116 .}$

Sauerland, Uli. 2014. Making fuzzy logic work for language. Semantics and Philosophy in Europe 7.

Sperber, Dan and Deirdre Wilson. 2008. A deflationary account of metaphors. In R. Gibbs, The Cambridge handbook of metaphor and thought, 84-106. Cambridge: Cambridge University Press.

Stanley, Jason. 2007. Language in context: Selected essays. Oxford: Clarendon Press.

Wilson, Deirdre and Robyn Carston. 20o6. Metaphor, relevance and the 'emergent property' issue. Mind \& Language 21(3): 404-433. 